\title{
Chromogenic In Situ Hybridization and p16/Ki67 Dual Staining on Formalin-Fixed Paraffin-Embedded Cervical Specimens: Correlation with HPV-DNA Test, E6/E7 mRNA Test, and Potential Clinical Applications
}

\author{
Roberta Zappacosta, ${ }^{1}$ Antonella Colasante, ${ }^{2}$ Patrizia Viola, ${ }^{2}$ Tommaso D'Antuono, \\ Giuseppe Lattanzio, ${ }^{2}$ Serena Capanna, ${ }^{2}$ Daniela Maria Pia Gatta, ${ }^{1}$ and Sandra Rosini ${ }^{1}$ \\ ${ }^{1}$ Cytopathology Unit, Experimental and Clinical Sciences Department, "G. d'Annunzio" University of Chieti-Pescara, \\ Via dei Vestini, 66100 Chieti, Italy \\ ${ }^{2}$ Surgical Pathology Unit, "SS Annunziata” Hospital, ASL2 Abruzzo, Chieti, Italy \\ Correspondence should be addressed to Roberta Zappacosta; zappacosta2@hotmail.com
}

Received 16 July 2013; Revised 20 September 2013; Accepted 30 September 2013

Academic Editor: Oronza Antonietta Botrugno

Copyright (C) 2013 Roberta Zappacosta et al. This is an open access article distributed under the Creative Commons Attribution License, which permits unrestricted use, distribution, and reproduction in any medium, provided the original work is properly cited.

\begin{abstract}
Although HPV-DNA test and E6/E7 mRNA analyses remain the current standard for the confirmation of human papillomavirus (HPV) infections in cytological specimens, no universally adopted techniques exist for the detection of HPV in formalin-fixed paraffin-embedded samples. Particularly, in routine laboratories, molecular assays are still time-consuming and would require a high level of expertise. In this study, we investigated the possible use of a novel HPV tyramide-based chromogenic in situ hybridization (CISH) technology to locate HPV on tissue specimens. Then, we evaluate the potential usefulness of $\mathrm{p} 16^{\text {INK4a }} / \mathrm{Ki}$ 67 double stain on histological samples, to identify cervical cells expressing HPV E6/E7 oncogenes. In our series, CISH showed a clear signal in $95.2 \%$ of the specimens and reached a sensitivity of $86.5 \%$. CISH positivity always matched with HPV-DNA positivity, while $100 \%$ of cases with punctated signal joined with cervical intraepithelial neoplasia grade 2 or worse (CIN2+). p16/Ki67 immunohistochemistry gave an interpretable result in 100\% of the cases. The use of dual stain significantly increased the agreement between pathologists, which reached 100\%. Concordance between dual stain and E6/E7 mRNA test was 89\%. In our series, both CISH and p16 $6^{\text {INK4a }} / \mathrm{Ki} 67$ dual stain demonstrated high grade of performances. In particular, CISH would help to distinguish episomal from integrated HPV, in order to allow conclusions regarding the prognosis of the lesion, while p16 ${ }^{\text {INK4a }} /$ Ki67 dual stain approach would confer a high level of standardization to the diagnostic procedure.
\end{abstract}

\section{Introduction}

HPV infection is recognised as the necessary cause of cervical intraepithelial lesions (CIN) and invasive squamous cell carcinoma (SSC). However, only a minority of viral infections ever results in neoplastic lesions. It is well known that the majority of HPV infections may be cleared by the immune system, and that certain high-risk (HR) HPV types (HPV 16, $18,31,33,45$, and 54 ) are significantly more common among high-grade lesions and carcinomas [1].

The most important factor in CIN progression is certainly the integration of HPV sequences into the host genome with the loss of E2 tumor suppressor gene. E2 physiologically regulates the expression of E6 and E7 oncogenes. There is consensus that integration is common in high-grade CIN and cancer, while it is infrequent or is lacking in lowgrade CIN. HPV integration, disrupting cell-cycle control and escaping immune system surveillance, would induce stochastic accumulation of genetic aberrations, leading to CIN progression.

Recently, a wide range of molecular techniques has been evaluated on cytological specimens, to improve cervical cancer screening strategies $[2,3]$. HPV-DNA test showed a high sensitivity in identifying CIN, but it still lacks clinical 
specificity, due to the high prevalence of transient infection [2]. E6/E7 mRNA test, targeting patients at higher risk of CIN progression, demonstrated to be more specific than DNA test in stratifying the risk for cancer development [4].

On tissue specimens, the ideal test for the detection of HPV has not been established yet, although different assays have been analyzed (i.e., PCR, in situ hybridization, ISH). Potential useful marker should target viral genome or related proteins (i.e., DNA, mRNA) or should identify host cell's products whose expression would be stimulated by HPV infection. In this context, immunohistochemical (IHC) localization of $\mathrm{p} 16^{\mathrm{INK} 4 \mathrm{a}}$ (henceforth $\mathrm{p} 16$ ) seems to represent one of the most widely investigated tool.

p16 is a tumor suppressor protein playing a crucial role in cell-cycle regulation. p16 prevents the phosphorylation of the retinoblastoma protein $(\mathrm{pRb})$ by inhibiting cyclindependent kinases CDK4 and CDK6. Physiologically, nonphosphorylated $\mathrm{pRb}$ binds the transcription factor E2F, thereby preventing E2F stimulation of cell progression into $S$ phase. The functional inactivation of $\mathrm{pRb}$ by HPV-E7 oncoprotein induces E2F factor release that becomes subsequently free to drive cell-cycle progression towards S phase.

All the above mentioned markers and technologies are a matter of controversy, each having their advantages and drawbacks.

PCR is considered the most effective method for HPVDNA detection, but some problems still exist in routinely practice: DNA extraction compromises the preservation of tissue architecture [5]; moreover, it requires a high of expertise and strict laboratory conditions, to avoid contaminations [6]. ISH is cheap and relatively easy to perform. It would permit the detection of HPV-DNA, as well as the preservation of histological pattern. On the other hand, ISH lacks in sensitivity (limit of 10-50 DNA copy/cell) [7, 8]. To by-pass this problem, a tyramide-based signal amplification kit, based on HPV chromogenic in situ (CISH) technology, has been developed [5].

p16 demonstrated to be useful as surrogate biomarker of HPV integration and E7 overexpression. However, pitfalls such as positive staining by nondysplastic cells would limit its clinical accuracy. Recently, a novel concept of biomarker based on the combination of p16 and Ki-67 detection in cervical cytology specimens (p16/Ki-67 double stain) has been proposed. Under physiological conditions, the coexpression of these proteins does not occur, since they typically induce opposite effects [6]. Simultaneous expression of both markers within the same cervical cell would indicate HPV-dependent deregulated cell cycle. Only limited results are available for p16/Ki67 assay $[6,9,10]$; all of these concerning its potential utility on cytological samples. To our knowledge, there are no data regarding the feasibility of p16/Ki67 double stain on histological specimens.

Basing on this background, in the first phase of this study, we aimed to analyze analytical and diagnostic accuracies of the novel CISH technology in detecting viral DNA and in identifying HPV physical status on formalin-fixed and paraffin-embedded tissue. To do that, $\mathrm{CISH}$ results were compared with results obtained from HPV-DNA test and HPV-mRNA test.
In the second phase, we assessed the potential usefulness of CINtec PLUS p16/Ki-67 double-stain immunohistochemistry (IHC) on histological samples with different degrees of dysplasia, to detect cervical lesions expressing E6/E7 HPV oncogenes.

\section{Materials and Methods}

2.1. Cervical Tissue Specimens Selection. This study was performed in agreement with the standards of the ethics review board of "SS Annunziata" Hospital and was approved by the Ethical Committees of “G. d'Annunzio" University, in accordance with the principles outlined in the Declaration of Helsinki of 1975.

From the electronic files of Surgical Pathology Department of "SS Annunziata" Hospital of Chieti, 926 cases of biopsy-proven squamous cervical lesion, obtained from January 2010 to July 2012, were retrospectively retrieved.

Among these casuistries, 154 cases met the following inclusion criteria:

(i) HPV-DNA test result by Hybrid Capture 2 (HC2), performed on liquid-based sample of exfoliated cells, collected from cervix immediately before colposcopydirected biopsy of the lesion;

(ii) result from mRNA testing, performed on residual cervical liquid-based cytological specimen.

Two pathologists independently reviewed haematoxylin and eosin (H\&E) stained slides and reported histological diagnosis according to the World Health Organization nomenclature and criteria as follows:

Cervical Intraepithelial Neoplasia grade 1, CIN1;

CIN grade 2, CIN2;

CIN grade 3, CIN3;

invasive squamous cell carcinoma (SSC).

Only cases reaching consensus in histological diagnosis were finally included in the study ( 63 formalin-fixed, paraffinembedded, FFPE).

A written informed consent was obtained from all the participants in the study, and corresponding FFPE specimens were subsequently taken. Identification codes were finally assigned to each case, in accordance with confidentiality standards.

\subsection{Laboratory Methods}

(i) Cervical Cytology. Cervicovaginal samples were collected from ecto-endocervix immediately before colposcopydirected biopsy. Cervical specimens were then transferred into PreservCyt cytology medium (Cytyc Corporation, Boxborough, MA) liquid and transported to Cytopathology Departments. Cytological vials were processed using ThinPrep 2000 (Hologic, Marlborough, MA, USA). Slides were next stained with Papanicolaou procedure.

(ii) HPV-DNA Test. After cytological slide preparation, an aliquot $(4 \mathrm{~mL}$ ) of each liquid-based cytological (LBC) sample, 
stored at RT, was removed to perform HPV-DNA testing by using the commercially available Hybrid Capture 2 system (HC2, Qiagen, Gaithersburg, MD), in accordance to manufacturer's protocol. HC2 detects oncogenic HPV types $(16,18$, $31,33,35,39,45,51,52,56,58,59$, and 68 ). HC2 reactions were read by a luminometer, which provided a relative quantification of each individual sample in comparison to the mean of a series of positive controls containing $1 \mathrm{pg} / \mathrm{mL}$ of HPV DNA (corresponding to $100,000 \mathrm{HPV}-16$ genomes $/ \mathrm{mL}$ or 5,000 HPV copies per reaction). The cut-off of 1 relative light unit (RLU) was used to classify a specimen as positive or negative. RLUs value in relation to control (RLU/CO) provided an estimation of the number of HPV-DNA copies of each sample (viral load). The RLU value of each individual sample was then recorded. According to RLU/CO values, HPV-DNA positive cases were arbitrarily categorized into three groups having "low viral load" (RLU/CO from 1.0 to 50.0 RLU/CO), “intermediate viral load” (RLU/CO from 50.1 to 100.00 RLU/CO), and "high viral load” (RLU/CO > 100).

(iii) HPV-mRNA Test. A second aliquot $(3 \mathrm{~mL})$ from each residual $\mathrm{LBC}$ specimen was transferred into a fresh $10 \mathrm{~mL}$ tube for nucleic acids extraction. After centrifugation, the supernatant was removed and the sample was transferred into a tube containing $2 \mathrm{~mL}$ Nuclisens Lysis Buffer (BioMèrieux, France). Next, magnetized silica dioxide particles were added to the lysate to initiate the nucleic acids isolation process. Finally, nucleic acids were eluted from the solid phase in $55 \mu \mathrm{L}$ of elution buffer and stored at $-20^{\circ} \mathrm{C}$ if not further processed immediately after extraction.

$15 \mu \mathrm{L}$ of nucleic acids was used to perform mRNA testing (Nuclisens EasyQ HPV, BioMèrieux, France), in accordance with the manufacturer's instructions.

mRNA testing is based on real-time nucleic acid sequence based amplification (NASBA) procedure, which utilizes molecular beacon probes labelled with 5-carboxyfluorescein (FAM) and Texas Red fluorochromes, at an isothermal temperature of $41^{\circ} \mathrm{C}$. The test identifies full-length E6/E7 mRNA from five high-risk carcinogenic HPV types $(16,18$, 31,33 , and 45). A fluorescent analyzer measured in real time the emission of the fluorescence from molecular beacon hybridized with amplified mRNA. As performance control, the human U1A mRNA from the small ribonucleoproteinspecific A protein has been used. Negative control reactions, consisting of all reagents except RNA, were performed at each run. mRNA testing was defined as positive if at least one of the five HPV genotypes detected by the test has been found [11].

(iv) Chromogenic In Situ Hybridization. Two serial sections were cut to a thickness of $4 \mu \mathrm{m}$, one for CISH investigation and one for p16/Ki67 dual-stain IHC. The extra sections cut before and after each tissue section were stained with $\mathrm{H} \& \mathrm{E}$ and used to evaluate the adequacy of each FFPE for the subsequent investigations.

Bond ready-to-use DNA CISH HPV protocol (Bond ready-to-use DNA ISH HPV Probe by Leica Biosystems, Newcastle Ltd, Newcastle, UK) able to detect 5 oncogenic HPV types (types 16, 18, 31, 33, and 54) was optimized for reproducible sensitive and background free usage. Slides were then processed using the Bond-Max automated slidestaining system (Leica Biosystems, Newcastle Ltd, Newcastle, UK). Finally, CISH sections were counterstained with haematoxylin. HPV-positive controls consisted of FFPE sections containing two sets of cells: CaSki cervical cancer cell line (containing 200 to 400 copies of HPV-DNA types 16 per cell) and HeLa cervical cancer cell line (containing 10 to 50 copies of HPV-DNA types 18 per cell). Thyroid tissue has been used as negative control, since in the literature we could not find any evidence for the presence of HPV.

Two pathologists independently evaluated CISH slides. $\mathrm{CISH}$ signals were determined for at least 10 high power fields. Nuclear peroxidase staining was considered a positive result for HPV-DNA. Positive CISH signal patterns were classified as follows: (1) diffuse (D), when nuclei were completely stained (indicative of episomal HPV); (2) punctated, when distinct dot-like intranuclear signals were noted (indicative of integrated HPV); (3) mixed, diffuses, and punctated (D/P) when both patterns are noted. (Figures 1(a)-1(c)).

(v) p16/Ki67 Dual Stain and p16 Stain. A commercial kit specifically designed for the simultaneous detection of p16 and Ki67 (CINtec PLUS Kit, Roche mtm laboratories, Heidelberg, Germany) was used, accordingly to the supplier's instructions and adapting the protocols for the use on histological samples. One section for each case was stained with p16/Ki67 dual test. A red chromogen marked Ki-67 expression within the nucleus and a brown chromogen marked cytoplasmic/nuclear p16 expression. Sample was scored as positive when the simultaneous expressions of both markers were revealed within the same cells. Cases without any doubleimmunoreactive cell were called negative.

Another section for each case was prepared for the immunohistochemical evaluation of p16 alone (clone E6/H4) using CINtec Histology Kit (Roche mtm laboratories, Heidelberg, Germany). After antigen retrieval, sections were incubated with mouse monoclonal anti-p16 (Lab Vision/NeoMarkers, Fremont, CA), with EnVision+ System HRP anti-mouse (Dako, Copenhagen, Denmark). Afterwards, diaminobenzidine chromogen (Dako, Copenhagen, Denmark) was applied and counterstaining with haematoxylin was performed. p16 overexpression was visualized as a brown colour precipitate within nucleus and cytoplasm. Expression of p16 in more than $10 \%$ of epithelial cells was regarded as a positive result.

For dual stain and p16 immunohistochemistry, positive and negative controls consisted of SCC of uterine cervix, with and without primary antibodies, respectively. All tissue slides plus controls for p16/Ki67 dual test were stained in a single session that was different from that of p16 alone. In both cases, Dako Autostainer (Dako, Copenhagen, Denmark) was used.

Both slide sets were subjected to two pathologists, which evaluated all cases blindly to all study results.

2.3. Statistical Analyses. By standard method authors calculated the prevalence of HPV-DNA, E6/E7 mRNA, p16/Ki67, and $\mathrm{CISH}$ positivities. Chi square or Fisher's exact test was used to assess the association between variables. Concordances between histopathological diagnosis and DNA test, 


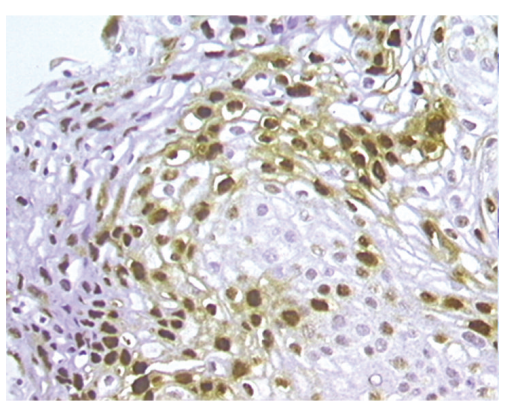

(a)

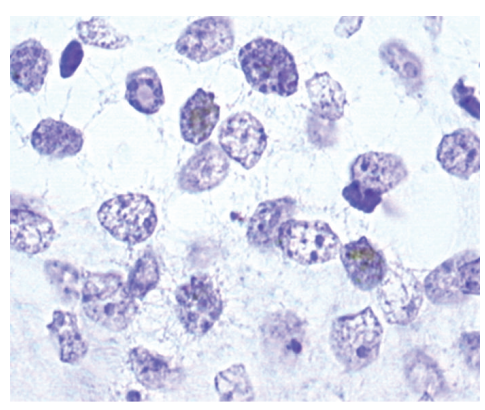

(b)

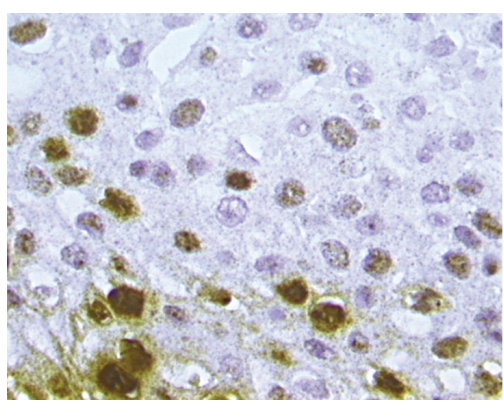

(c)

FIGURE 1: CISH positive signals. Diffuse pattern, where nuclei are completely stained ((a), 20x magnification). Punctated pattern in invasive squamous cervical cancer: distinct dot-like intranuclear signals were noted within cells infiltrating the stroma ((b), 100x magnification). Mixed patterns, where both diffuse and punctated signals are noted ((c), 40x magnification).

mRNA test, and CISH were calculated by Kappa statistics. According to the criteria of Lands and Koch, the $K$ values were divided into six scales of strength of agreement: poor $(<0.00)$, slight $(0.00-0.20)$, fair $(0.21-0.40)$, moderate $(0.41-$ $0.60)$, substantial (0.61-0.80), or almost perfect (0.81-1.00) [12]. Chi square for trend (Cochran-Armitage test) was calculated to assess the trend of CISH results in relation with the severity of cervical disease.

Accuracy parameters (sensitivity and specificity) of each test separately as well as the comparison of accuracy parameters between tests were assessed by receiver operating characteristic analysis. Histological diagnosis was regarded as the gold standard and CIN2+ lesion was considered as the worse outcome. To do that, histological results were dichotomized into CIN2+ (including CIN2, CIN3, and SCC) and less than CIN2 (CIN2-, including CIN1). Areas under the receiver operating characteristic (ROC) curves and $95 \%$ confidence intervals (CI) were estimated to assess differences between test performances [13] and McNemar test was used for statistical significance.

Correlation between CISH signal patterns and HPV viral load categories was evaluated by Cochran-Armitage trend test.

Statistical analyses were performed by using SPSS software (SPSS for Windows, Inc., Chicago, IL), version 15.0. In all analyses, probability values $P$ less than 0.05 were regarded as significant.

2.4. Results. A series of cervical FFPE from sixty-three patients (mean age $34 \pm 8$ years, median 33 years, range 2163) were included in the study. Among these cases, 25 were diagnosed as CIN1, 16 as CIN2, 21 as CIN3, and 1 as SCC.

Summary of results from histological diagnosis, HPVDNA and mRNA tests, HPV viral load, CISH, and p16/Ki67 dual stain from each case included in the study are reported in Table 1.

Molecular Tests. HPV-DNA positivity was detected in 60 of the $63(95.2 \%)$ cytological samples. Among these, $65 \%(N=$ $39 / 60$ ) showed CIN2+ lesions in histological specimens. A positive DNA test result conferred a $\geq \mathrm{CIN} 2+$ odds ratio
(OR) risk of 3.2 (95\% CI: $0.4-26) .4 .8 \%(N=3 / 63)$ of women resulted HPV-DNA negative. Overall percent agreement between DNA testing test and histological diagnosis was $61.9 \%$ (Cohen's kappa value: $0.06, P<0.05$ ). E6/E7 mRNA positivity was detected in $71.4 \%(N=45 / 63)$ of cytological cases; among these, 36 (80\%) were CIN2+. Within the $18 \mathrm{mRNA}$ negative cases, $16(88.9 \%)$ were confirmed as CIN2-. mRNA test results were associated to CIN2+ diagnosis with a OR = 32 (95\% CI: 7-144). Overall percent agreement between mRNA testing and histological diagnosis was $82.5 \%$ (Cohen's kappa value: $0.62, P<0.0001$ ).

Diagnostic performances of both DNA and mRNA tests are represented in Table 2. mRNA test improved specificity of DNA testing. Difference was statistically significant $(\mathrm{McNe}-$ mar test, $P<0.01$ ).

CISH Results. CISH showed a clear signal in $95.2 \%(N=$ $60 / 63$ ) of the specimens. Invalid result has been found in $4.8 \%(N=3 / 63)$ of the cases, due to unclear and weak signal. The rate of positive results was $73 \%(N=46 / 63)$. Among these, 30.4\% $(N=14)$ were CIN1, 30.4\% $(N=14)$ were CIN2, and 37\% $(N=17)$ were CIN3. The unique case of SCC showed CISH positivity. Negativity has been found in $22.2 \%(N=14 / 63)$ of the cases. Table 3 shows details of the distribution of CISH signal patterns and their correlation with histological diagnosis. As expected, CISH showed a clear punctated signal pattern in both HPV positive cell lines, whereas no signal was detected in thyroidal tissue. Nonspecific background binding has never been seen among the 60 cases which were considered as valid cases. Notably, about two-thirds of diffuse pattern were associated with CIN1, while the unique case of SCC displayed a punctated pattern. Differences were statistically significant $(P<0.01)$. Dichotomizing histological diagnosis and considering only CISH-positive results, diffuse pattern has been found in 64.3\% $(N=9 / 14)$ of CIN2- and 3.1\% $(N=1 / 32)$ of CIN2+. All cases of punctated pattern have been found in CIN2+, as well as $68.8 \%$ of mixed patterns. The proportion of punctated pattern increased with the severity of cervical lesion (Cochran-Armitage test for trend $P<0.0001$ ) (Figure 2). 
TABLE 1: Summary of results from histological diagnosis, HPV-DNA and mRNA tests, HPV viral load, CISH, and p16/Ki67 dual stain.

\begin{tabular}{|c|c|c|c|c|c|c|c|}
\hline Case & $\begin{array}{l}\text { Histological } \\
\text { diagnosis }\end{array}$ & $\begin{array}{l}\text { HR HPV-DNA } \\
\text { test result }\end{array}$ & $\begin{array}{c}\mathrm{HC} 2 \\
\text { viral load } \\
(\mathrm{RLU} / \mathrm{CO})^{*}\end{array}$ & $\begin{array}{l}\text { E6/E7 mRNA test } \\
\text { result }\end{array}$ & $\begin{array}{c}\text { Type specific } \\
\text { mRNA test result** }\end{array}$ & $\begin{array}{c}\mathrm{HPV} \mathrm{CISH} \\
\text { signal }\end{array}$ & $\begin{array}{c}\text { P16/Ki67 } \\
\text { Dual stain result }\end{array}$ \\
\hline 1 & CIN1 & Positive & 63.5 & Negative & - & $P$ & Negative \\
\hline 2 & CIN1 & Positive & 516.61 & Negative & - & $D$ & Negative \\
\hline 3 & CIN3 & Positive & 15.79 & Negative & - & Negative & Positive \\
\hline 4 & CIN2 & Positive & 146.77 & Positive & 16 & $D / P$ & Positive \\
\hline 5 & CIN2 & Positive & 1283.97 & Negative & - & $D / P$ & Positive \\
\hline 6 & CIN1 & Positive & 298.55 & Negative & - & $D$ & Negative \\
\hline 7 & CIN3 & Positive & 1529.35 & Positive & 16,31 & $D / P$ & Positive \\
\hline 8 & CIN3 & Positive & 2207.8 & Positive & 31 & $D / P$ & Positive \\
\hline 9 & CIN1 & Positive & 2.06 & Negative & - & $D$ & Negative \\
\hline 10 & CIN2 & Positive & 216.93 & Positive & 31 & $D / P$ & Positive \\
\hline 11 & CIN1 & Positive & 6.7 & Negative & - & Negative & Negative \\
\hline 12 & CIN2 & Positive & 84.32 & Positive & 31 & Negative & Positive \\
\hline 13 & CIN3 & Positive & 10.56 & Positive & 16 & $D / P$ & Positive \\
\hline 14 & CIN3 & Positive & 338.02 & Positive & 16 & $D / P$ & Positive \\
\hline 15 & CIN1 & Positive & 1211.84 & Positive & 33 & $D / P$ & Positive \\
\hline 16 & CIN3 & Positive & 3.3 & Positive & 16 & $P$ & Positive \\
\hline 17 & CIN1 & Positive & 155.79 & Positive & 16 & $D$ & Negative \\
\hline 18 & CIN1 & Positive & 28.81 & Negative & - & Negative & Positive \\
\hline 19 & CIN2 & Positive & 34.82 & Negative & - & $D$ & Positive \\
\hline 20 & CIN3 & Positive & 913.36 & Negative & - & $D$ & Positive \\
\hline 21 & CIN2 & Positive & 1536.02 & Positive & 16 & $P$ & Negative \\
\hline 22 & CIN2 & Positive & 107.22 & Positive & 18,45 & $D / P$ & Positive \\
\hline 23 & CIN1 & Positive & 75.86 & Negative & - & $D / P$ & Negative \\
\hline 24 & CIN2 & Positive & 675.75 & Positive & 16 & $D / P$ & Positive \\
\hline 25 & CIN2 & Positive & 596.84 & Positive & 16 & $D / P$ & Positive \\
\hline 26 & CIN3 & Positive & 1914.17 & Positive & 16 & $D / P$ & Positive \\
\hline 27 & CIN2 & Positive & 570.26 & Positive & 16,45 & $D / P$ & Positive \\
\hline 28 & CIN1 & Positive & 783.56 & Negative & - & $D$ & Negative \\
\hline 29 & CIN1 & Negative & - & Negative & - & Negative & Negative \\
\hline 30 & CIN3 & Positive & 3.93 & Positive & 16 & $P$ & Positive \\
\hline 31 & CIN3 & Positive & 968.56 & Positive & 31 & $D / P$ & Positive \\
\hline 32 & CIN1 & Positive & 926.05 & Positive & 31 & Invalid & Negative \\
\hline 33 & CIN2 & Negative & - & Positive & 31 & Invalid & Positive \\
\hline 34 & CIN1 & Positive & 45 & Negative & - & $D$ & Negative \\
\hline 35 & CIN3 & Positive & 117 & Positive & 16 & $D / P$ & Positive \\
\hline 36 & CIN1 & Positive & 237.48 & Positive & 16 & Invalid & Negative \\
\hline 37 & CIN1 & Positive & 5.31 & Positive & 18,31 & Negative & Negative \\
\hline 38 & CIN1 & Positive & 204.42 & Positive & 45 & Negative & Negative \\
\hline 39 & CIN3 & Positive & 663.26 & Positive & 33 & Negative & Positive \\
\hline 40 & CIN3 & Positive & 38.61 & Positive & 16 & Negative & Positive \\
\hline 41 & CIN1 & Positive & 10.11 & Positive & 33 & $D / P$ & Positive \\
\hline 42 & CIN1 & Positive & 137.88 & Negative & - & $D$ & Negative \\
\hline 43 & CIN3 & Positive & 6.21 & Positive & 16 & Negative & Positive \\
\hline 44 & CIN2 & Positive & 2663.26 & Positive & 31 & $D / P$ & Positive \\
\hline 45 & SCC & Positive & 1549.74 & Positive & 33 & $D / P$ & Positive \\
\hline 46 & CIN2 & Positive & 894.17 & Positive & 16 & $P$ & Positive \\
\hline
\end{tabular}


TABle 1: Continued.

\begin{tabular}{|c|c|c|c|c|c|c|c|}
\hline Case & $\begin{array}{c}\text { Histological } \\
\text { diagnosis }\end{array}$ & $\begin{array}{l}\text { HR HPV-DNA } \\
\text { test result }\end{array}$ & $\begin{array}{c}\mathrm{HC} 2 \\
\text { viral load } \\
(\mathrm{RLU} / \mathrm{CO})^{*}\end{array}$ & $\begin{array}{l}\text { E6/E7 mRNA test } \\
\text { result }\end{array}$ & $\begin{array}{c}\text { Type specific } \\
\text { mRNA test result** }\end{array}$ & $\begin{array}{l}\mathrm{HPV} \mathrm{CISH}^{\dagger} \\
\text { signal }\end{array}$ & $\begin{array}{c}\text { P16/Ki67 } \\
\text { Dual stain result }\end{array}$ \\
\hline 47 & CIN1 & Positive & 20.47 & Negative & - & Negative & Negative \\
\hline 48 & CIN1 & Positive & 26.23 & Negative & - & Negative & Negative \\
\hline 49 & CIN1 & Positive & 787.16 & Positive & 31 & Negative & Negative \\
\hline 50 & CIN1 & Positive & 676.46 & Positive & 16 & $D / P$ & Negative \\
\hline 51 & CIN3 & Positive & 2.36 & Positive & 16 & $P$ & Positive \\
\hline 52 & CIN3 & Positive & 1.45 & Positive & 18 & $P$ & Positive \\
\hline 53 & CIN3 & Positive & 111.95 & Positive & 16,18 & $D / P$ & Positive \\
\hline 54 & CIN3 & Positive & 544.41 & Positive & 16 & $D / P$ & Positive \\
\hline 55 & CIN2 & Positive & 663.21 & Positive & 16 & $D / P$ & Positive \\
\hline 56 & CIN1 & Negative & - & Negative & - & Negative & Negative \\
\hline 57 & CIN2 & Positive & 1569.56 & Positive & 18 & $D / P$ & Positive \\
\hline 58 & CIN3 & Positive & 758.66 & Positive & 16 & $D / P$ & Positive \\
\hline 59 & CIN2 & Positive & 130.13 & Positive & 16 & $D / P$ & Positive \\
\hline 60 & CIN3 & Positive & 87.01 & Positive & 16 & $P$ & Positive \\
\hline 61 & CIN3 & Positive & 968.56 & Positive & 31 & $D / P$ & Positive \\
\hline 62 & CIN2 & Positive & 6.21 & Positive & 16 & $P$ & Positive \\
\hline 63 & CIN3 & Positive & 24.01 & Positive & 16 & $P$ & Positive \\
\hline
\end{tabular}

${ }^{*}$ Relative light unit in relation to control (RLU/CO).

${ }^{* *}$ HPV genotype(s) detected by Nuclisens EasyQ HPV mRNA test.

${ }^{\dagger} \mathrm{D}$ : diffuse; P: punctated; D/P: mixed diffuse/punctated.

TABle 2: Diagnostic performances of HPV-DNA test (HC2) and E6/E7 mRNA test.

\begin{tabular}{lcc}
\hline \multirow{2}{*}{ Molecular testing } & \multicolumn{2}{c}{$\begin{array}{l}\text { Diagnostic performances }\left(95 \% \mathrm{CI}^{*}\right) \\
\text { Sensitivity }\end{array}$} \\
\hline HPV-DNA test & $97.4 \%(85.1-100)$ & $8 \%(1.2-26)$ \\
HPV-mRNA test & $90.7 \%(81.6-99.4)$ & $64 \%(44.4-79.7)$ \\
\hline
\end{tabular}

${ }^{*}$ Confidence intervals (CI).

Sensitivity and specificity of CISH analysis were $86.5 \%$ (95\% CI: 71.4-94.4) and 39.1\% (95\% CI: 22.2-59.3), respectively. A positive $\mathrm{CISH}$ result conferred $\mathrm{a} \geq \mathrm{CIN} 2+$ risk (OR) of 4.11 (95\% CI: 2-13.9).

CISH results were assessed against HPV-DNA test (Figure 3). All CISH-positive cases also resulted HPV-DNA positive. Among HPV-DNA positive patients, $76.7 \%(N=$ 46/60) were CISH positive. Within CISH-negative cases, 85.7\% $(N=12 / 14)$ were HPV-DNA positive, while $14.3 \%$ $(N=2 / 14)$ were HPV-DNA negative $(P=.001)$. Overall percent agreement between CISH and DNA test was $80 \%$ $(k=0.20, P<0.05)$.

CISH results were also assessed against HPV E6/E7 mRNA expression. Among mRNA+ cases, 77.8\% $(N=$ $35 / 45)$ were CISH positive. Of those, $2.9 \%(N=1 / 35)$ showed a diffuse pattern, $71.4 \%(N=25 / 35)$ a mixed pattern, and $25.7 \%(N=9 / 35)$ a punctated pattern. Among the $11 \mathrm{mRNA} / \mathrm{CISH}+$ cases, only 2 cases $(18 \%)$ demonstrated a punctated pattern $(P<0.0001)$. Overall percent agreement between CISH and mRNA test was 70\% $(k=0.24, P<0.05)$.

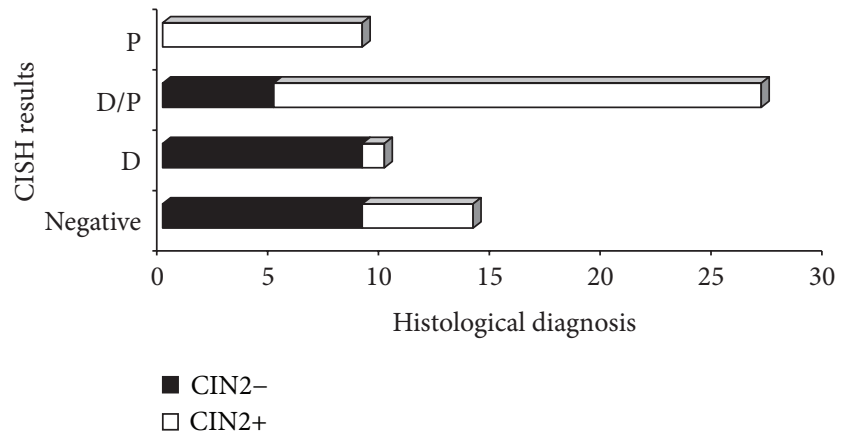

FIGURE 2: Correlation between CISH results and histological diagnosis $(\mathrm{P}<0.0001)$. $\mathrm{P}$ : punctate pattern; $\mathrm{D} / \mathrm{P}$ : diffuse and punctated (mixed) pattern; D: diffuse pattern. CIN2+: Cervical intraepithelial neoplasia grade 2 or greater (including CIN2, CIN3, and invasive squamous cell carcinoma); CIN2-: less than Cervical Intraepithelial Neoplasia grade 2 (including CIN1 and negative for dysplasia.

Since HPV-DNA test is currently considered the most reliable method to detect papillomavirus infection in both cytological and histological samples, the performances of CISH and mRNA test were compared to HPV-DNA test performance. DNA testing achieved an area under the curves (AUC) of 0.53 (95\% CI, 0.4-0.65) $\mathrm{CISH}$ and of 0.64 (95\% CI, 0.5-0.75) and mRNA testing of 0.79 (95\% CI, 0.67-0.89) (Figure 4). Difference between HPV-DNA test and mRNA test was statistically significant $(P<0.0001)$, while difference between RNA testing and $\mathrm{CISH}$ did not reach significance $(P=0.06)$. 
TABLE 3: Association between CISH signal patterns and grading of cervical lesions $(P<0.01)$.

\begin{tabular}{|c|c|c|c|c|c|}
\hline \multirow{2}{*}{ CISH result } & \multicolumn{4}{|c|}{ Number of cases (\%) } & \multirow{2}{*}{ Total } \\
\hline & CIN1 & CIN2 & CIN3 & SCC & \\
\hline Invalid & $2(8)$ & $1(6.3)$ & 0 & 0 & $3(4.8)$ \\
\hline Negative & $9(36)$ & $1(6.3)$ & $4(19)$ & 0 & $14(22.2)$ \\
\hline Diffuse & $9(36)$ & $1(6.3)$ & 0 & 0 & $10(15.9)$ \\
\hline Diffuse-punctated & $5(20)$ & $11(68.7)$ & $11(52.4)$ & 0 & $27(42.8)$ \\
\hline Punctated & 0 & $2(12.4)$ & $6(28.6)$ & $1(100)$ & $9(14.3)$ \\
\hline Total & $25(39.7)$ & $16(25.4)$ & $21(33.3)$ & $1(1.6)$ & 63 \\
\hline
\end{tabular}



FIgURE 3: Correlation between CISH signal and results from molecular tests $(k=0.20, P<0.05)$.



FIGURE 4: Receiving operating characteristic curves (ROC), comparing CISH, HPV-DNA test, and E6/E7 mRNA diagnostic performances. The red line indicates a reference threshold with area under the ROC curve of 0.5 .

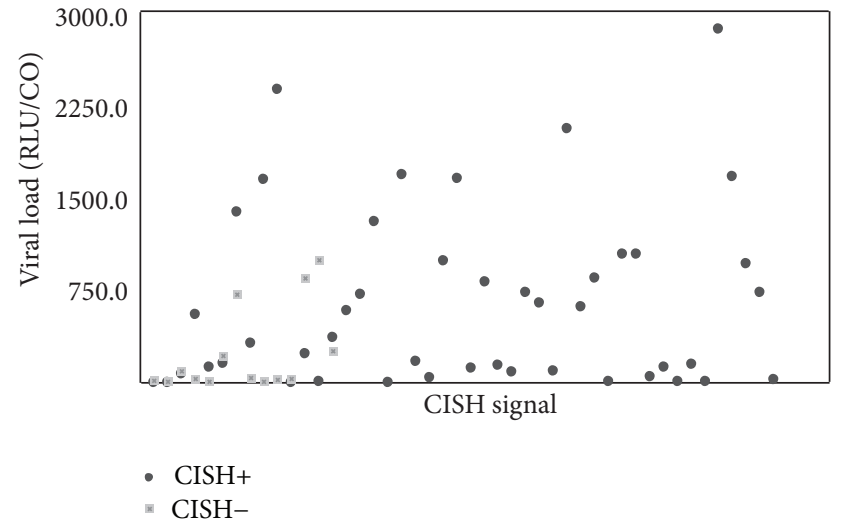

FIGURE 5: Correlation between CISH signal and HPV viral load, as detected by HC2 test $(P=0.01)$. RLU/CO value provided an estimation of the number of HPV-DNA copies of each sample. RLU/CO: ratio between relative light units and control.

CISH Results and HPV Viral Load. Among cytological samples testing HPV-DNA positive, the mean of viral loads was $502.9 \pm 620.5 \mathrm{RLU} / \mathrm{CO}$, the median being $155.79 \mathrm{RLU} / \mathrm{CO}$ (range 1.45-2663.29 RLU/CO).

Considering the categories of viral load values as described in Section 2, 31.7\% $(N=19 / 60)$ of the cases showed low viral load, $6.7 \%(N=4 / 60)$ intermediate load, and $61.6 \%$ $(N=37 / 60)$ high viral load. The rate of CISH positivity has been found to be lower in cases with low viral load level (58\%) than in those with intermediate $(75 \%)$ and high $(86.6 \%)$ load levels (Cochran-Armitage trend test, $P=0.01$ ) (Figure 5).

Correlation between CISH punctate signal pattern and viral load categories showed that the rate of this pattern was higher in specimens with low viral loads than in those having intermediate or high loads (Fisher exact test, $P=0.05$ ).

p16 and p16/Ki67 Dual Stain Analysis. Both p16 immunohistochemistry and p16/Ki67 analysis were performed on the entire FFPE series.

A positive p16 result was defined as a diffuse moderateto-strong cytoplasmic and nuclear staining. There was no difference in the intensity of staining between the different epithelial layers. Brown staining of normal metaplastic or endocervical cells was considered as negative p16 test.

When the diagnosis of cervical lesion was categorized into four, that is, CIN1, CIN2, CIN3, and SCC, a complete concordance for all the two observers was obtained in 32 


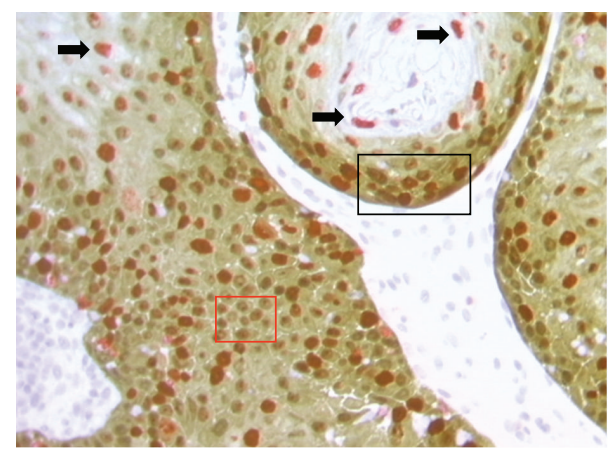

Figure 6: p16/Ki67 dual stain (40x magnification). Red square: brown chromogen marked cytoplasmic/nuclear p16 expression. Black arrows: red chromogen marked Ki-67 expression within nuclei. Black square: simultaneous expressions of both markers were revealed within the same cells.

cases (51\%), including $8 \mathrm{CIN} 1,11 \mathrm{CIN} 2,12 \mathrm{CIN} 3$, and 1 SCC $(k=0.06)$. The lower agreement was observed for CIN1 diagnosis, the higher for SCC $(P=0.08)$ (Table 4$)$. Sensitivity and specificity of p16 IHC were 96.4 (95\% CI: 85.1-100) and 100\% (95\% CI: 83.9-100), respectively.

Considering p16/Ki67 dual stain immunohistochemistry (Figure 6), all 63 histological samples gave interpretable results. p16 expression was observed in 48 of 63 cases (76.2\%). Ki67 expression has been found in all histological specimens. Particularly, 13/25 CIN1 cases (52\%) showed weak Ki67 expression in the basal layer of cervical epithelium. The remaining $12 \mathrm{CIN1}$ cases showed strong nuclear Ki67 expression in the lower part of the epithelium (one-third), associated with cytoplasmic expression of p16 within the same cells. As the CIN grade was higher, stronger Ki67 expression was observed, particularly in $87.5 \%$ of CIN2 $(N=$ 14/16) cases (within two-third of cervical epithelium) and in $100 \%$ of CIN 3 cases (within the three-third of the epithelium). Expression level of p16 positively correlated with that of Ki67 $(P<0.01)$. In the unique case of SCC, strong dual-stain positivity has been also shown by neoplastic cells infiltrating the stroma.

The use of p16/Ki67 IHC significantly improved consensus among pathologists, which reached $100 \%(k=1)$. Sensitivity and specificity of dual stain were 100\% (95\% CI: 88.8-100) and 84\% (95\% CI: 64.6-94.1), respectively.

Since in cervical tissue p16 is considered a surrogate biomarker of HPV-E7 expression, we correlated both p16 and p16/Ki67 staining results with HPV-E6/E7 status, as determined by mRNA test (Table 5). p16 expression was observed in $77.8 \%(N=35 / 45)$ of mRNA-positive cases. Among mRNA-negative cases, p16 showed no immunoreactivity in $88.9 \%(N=16 / 18)$ of patients. Concordance between p16 and E6/E7 mRNA test was $81 \%(k=0.59)$.

Dual stain positivity has been found in $88.9 \%(N=$ 40/45) of mRNA-positive patients, negativity being detected in $88.8 \%(N=16 / 18)$ of mRNA-negative cases. Concordance between p16/Ki67 dual stain and mRNA test was $89 \%(k=$ $0.74)$.

Concordance between dual stain and CISH (punctated and mixed pattern) was $83.3 \%(k=0.64)$.

\section{Discussion}

Although HPV-DNA and E6/E7 mRNA tests still remain the current standards for the confirmation of HPV infections in cytological specimens, no consensus exists about technology that should be used for the detection of Papillomavirus in formalin-fixed paraffin-embedded samples [14]. This fact presents the clinicians with the dilemma of selecting the more suitable method. Molecular techniques (such as PCR) certainly represent the gold standard method, reaching a sensitivity of 1 DNA copy/cell [14]. However, DNA extraction requires trained laboratory personnel and is still highly timeconsuming and labour intensive for routine application. In addition, to detect HPV-DNA, a wide range of consensus primers, such as MY09/11, PGMY09/11, GP5+/6+, and SPF, are available [15]. Amplification with each of these primers provides amplification products of different sizes, thus providing different levels of sensitivity in viral detection. Particularly on FFPE material, because of the damaged and fragmented DNA, it is possible that the use of these primers could reach a high rate of false negative results [14]. It has been already shown that the maximum accuracy of PCR is obtained using fresh frozen tissues [16].

All consensus PCR primers for the detection of HPVDNA would target $\mathrm{L} 1$ region. This region is deleted when HPV-DNA is integrated into the host cell genome [17]. So, when HPV integration would occur, PCR should probably give false negative results.

Finally, due to its high sensitivity, PCR would detect HPV infection without any correlation with the prognosis of cervical lesion.

ISH is certainly less sensitive than PCR [18], but the visualization of HPV-DNA signals within nuclei of cervical lesions could offer both detection and localization of HPVDNA without damage of morphology. In addition, ISH helps to distinguish between episomal HPV from integrated one, the last being the necessary condition for neoplastic progression [19]. However, the low analytic sensitivity of ISH, ranging from 10 to 50 copy/cell, would be a weakness in case of high-grade cervical lesions in which, due to the frequent integration status of HPV, DNA copy number is usually less than 50 copy/cell. [7, 8]. Then, the choice of ISH technique would be extremely important. Non-tyramidebased methods showed too low sensitivity rate $[20,21]$. On the other hand, the higher sensitivity of tyramide-based ISH could lead to interpretation bias, especially due to nonspecific staining [22]. Hence, our aim is to analyse the performances of an optimized chromogenic ISH tyramidebased biotin-free assay.

In our FFPE series, sensitivity of CISH was about $87 \%$, higher if compared to series using non-tyramide-based methods $[19,22,23]$. CISH positive cases were characterized by a clear background. The rate on invalid results was very low (4.8\%) and due to scant FFPE specimens. CISH positivity always matched with HPV-DNA positivity. 20\% of HPVDNA positive cases demonstrated negativity at $\mathrm{CISH}$ analysis. The latter data may probably be due to the limited number of oncogenic genotypes detected by CISH probe (HPV 16, $18,31,33$, and 54), in comparison to those detected by HC2. 
TABLE 4: p16 immunostaining: interobserver agreement within histological categories of cervical lesions.

\begin{tabular}{|c|c|c|c|c|c|}
\hline \multirow{2}{*}{ p16 interobservers agreement } & \multicolumn{5}{|c|}{ Histological diagnosis } \\
\hline & CIN1 (\%) & CIN2 (\%) & CIN3 (\%) & SCC (\%) & Total \\
\hline Positive & $8(32)$ & $11(68.8)$ & $12(57.1)$ & $1(100)$ & $32(50.8)$ \\
\hline Negative & $17(68)$ & $5(31.2)$ & $9(42.9)$ & 0 & $31(49.2)$ \\
\hline Total & $25(39.7)$ & $16(25.4)$ & $21(33.3)$ & $1(1.6)$ & 63 \\
\hline
\end{tabular}

TABLE 5: Correlation between p16 and p16/Ki67 immunohistochemistry and E6/E7 mRNA test.

\begin{tabular}{|c|c|c|c|}
\hline \multirow{2}{*}{ Immunohistochemistry } & \multicolumn{3}{|c|}{ E6/E7 mRNA test } \\
\hline & Positive (\%) & Negative (\%) & Total \\
\hline p16 positive & $35(77.8)$ & $2(11.1)$ & $37(58.7)$ \\
\hline p16 negative & $10(22.2)$ & $16(88.9)$ & $26(41.3)$ \\
\hline Total & 45 & 18 & 63 \\
\hline p16/Ki67 dual stain positive & $40(88.9)$ & $2(11.1)$ & $42(66.7)$ \\
\hline p16/Ki67 dual stain negative & $5(11.1)$ & $16(88.8)$ & $21(33.3)$ \\
\hline Total & 45 & 18 & 63 \\
\hline
\end{tabular}

Anyhow, HPV types identified by CISH would represent five of the six most oncogenic genotypes, the sixth being HPV-45 $[1,24-26]$.

It is now well known that HPV integration is common in CIN2+ lesions while is uncommon or absent in CIN1 [27]. Studies on cervical carcinomas and SCCs cell lines demonstrated that oncogenic E6/E7 oncogenes are frequently overexpressed during HPV integration [27]. In our study, $100 \%$ of cases with punctate signal matched with CIN2+, while $94.7 \%$ of CIN2+ showed E6/E7 oncogenic expression (E6/E7 mRNA positivity). Percent agreement between CISH and mRNA test was high. Thus, we may conclude that CISH punctate signal confirmed as a sign of viral integration [18]. The only two CISH+/mRNA cases were probably due to HPV genotype 54 , detected by CISH but not detected by mRNA test.

In our series, when present, diffuse signal has been detected within cells of the mid/superficial layers. This pattern was mainly associated with CIN2-/mRNA negative cases and confirmed as a marker of productive HPV infections. Diffuse and punctate signals within the same lesion have also been found. This mixed pattern was associated with CIN2+ in $81.5 \%$ of the cases. This fact would be due to the polyclonal nature of cervical intraepithelial lesions. The unique case of infiltrating SCC showed punctate signal only, confirming the monoclonality of invasive neoplasia.

Although our cohort encompassed a limited number of cases, our preliminary results underline the usefulness of the tyramide-based CISH protocol which we used. This technology does not suffer of nonspecific background, simultaneously allowing the detection of HPV genome within morphological context. In addition, the use of a chromogen in alternative to fluorescence revealed to be more convenient for routine purpose, given the wide availability of light microscopy in pathology settings. Finally, CISH protocol could prove helpful also during the followup of patients with cervical lesions, as a feasible alternative to HPV-DNA and E6/E7 mRNA tests on FFPE specimens.

Recent researches on cervical cancer widely analysed biomarkers resulting associated with the various stages of HPV infection [3]. One of these strongly related to transforming HPV infection would be p16. Overexpression of p16 seems to increase with increasing degree of cervical lesion [28, 29]. A meta-analysis on p16 immunostaining on cytological and histological cervical specimens estimated that $2 \%$ of normal tissues and $38 \%$ of CIN1 showed diffuse staining, compared with $68 \%$ of CIN2 and $82 \%$ of CIN3 [30]. p16 immunostaining demonstrated to be cheap and easy to perform in pathology laboratories. The semiquantitative scoring system described by Klaes et al. [31], is actually the most widely used approach for the evaluation of this marker on histological specimens. However, estimation of results is often based on colorimetric and morphological criteria which are often subjective. This lack of standardization would make the use of this biomarker somehow difficult [30]. The assessment of p16 staining can be also hampered by false positive results $[32,33]$. Endometrial, metaplastic, and endocervical cells, as well as tubo-endometrioid metaplasia would stain p16-positive [34], since a non-HPV dependent p16 expression pathway may also exist $[4,35]$. For all the above mentioned reasons, there would be considerable reluctance among histopathologists to incorporate p16 IHC into routine gynae-pathology. Specifically, in our series the evaluation of p16 immunoreactivity generated a great variability in the interpretation and reached a low agreement level (51\%).

Nowadays, there is a considerable interest in the evaluation of the combination p16/Ki67, which would allow to differentiate dysplastic cells from nondysplastic ones, and meaningless HPV infection from transforming ones. In the present study, we performed p16/Ki67 dual stain immunohistochemistry on FFPE series of specimens encompassing all grades of morphological abnormalities. In our experience, 
this genotype-independent method has proved to be feasible and highly efficient in producing valid results. Even though in a limited series, dual stain results were always unequivocal. Moreover, inter-observer agreement was highest (100\%), since only cells simultaneously showing p16/Ki67 expression have been considered as positive, irrespective of morphology. Finally, in our series dual stain improved specificity of p16 alone.

In this setting, $98 \%$ of CIN2+ stained mRNA positive, while $100 \%$ stained p16/Ki67-positive. The only invasive cancer showed dual stain and E6/E7 mRNA positivity. It seems likely that dual stain positive/mRNA positive CIN2+ could represent cervical lesions at higher risk of progression toward invasive cancer [36]. This fact could not be determined in the present setting, since all CIN2+ lesions were surgically removed [37].

\section{Conclusion}

$\mathrm{HPV}$ are recognized as a necessary cause of CIN, but only a minority of HPV infections even results in cervical lesions. Although the majority of infections may be cleared by immune system, integration of HPV sequence into the host genome may induce CIN progression. The detection of HPV genome within cervical lesions and the assessment of its physical status are then crucial in prognostic terms.

To the authors' knowledge, this is the first study evaluating the novel HPV tyramide-based CISH technology and the innovative CINtec PLUS p16/Ki-67 double stain immunohistochemistry on histological tissues, as well as the first investigation comparing both methods to molecular tests actually considered as the gold standards for HPV detection.

Molecular assays may be expensive and require a high level of expertise, which are often difficult to reach in routinely laboratory. Although larger studies are needed, our data demonstrate the usefulness of CISH and p167Ki67 immunostaining in surgical pathology settings.

In particular, CISH could be a feasible method to localize HPV genome on paraffin-embedded specimens. This technology would help to distinguish episomal from integrated $\mathrm{HPV}$, thus allowing conclusions regarding the prognosis of the lesion. Likewise, the genotype-independent p16/Ki67 dual staining approach, which demonstrated greater efficacy than p16 alone, would confer a higher level of standardization to the diagnostic procedure.

Finally, due to their strong correlation with tests which are currently considered the standards for HPV detection in cytological specimens, both CISH and dual stain technologies would be considered a viable potential alternative to molecular assays in the evaluation of the biology of cervical lesions.

Nevertheless, these preliminary data need to be confirmed in a larger clinical cohort.

\section{Disclosure}

The authors specify that this paper has not been published, submitted, or accepted for publication elsewhere.

\section{Conflict of Interests}

All the authors declare that they have no conflict of interests.

\section{Authors' Contribution}

Roberta Zappacosta and Antonella Colasante contributed equally to this paper.

\section{References}

[1] R. Zappacosta and S. Rosini, "Cervical cancer screening: from molecular basis to diagnostic practice, going through new technologies," Technology in Cancer Research and Treatment, vol. 7, no. 3, pp. 161-174, 2008.

[2] J. Cuzick, C. Clavel, K. Petry et al., "Overview of the European and North American studies on HPV testing in primary cervical cancer screening," International Journal of Cancer, vol. 119, no. 5, pp. 1095-1101, 2006.

[3] J. Cuzick, M. Arbyn, R. Sankaranarayanan et al., "Overview of human papillomavirus-based and other novel options for cervical cancer screening in developed and developing countries," Vaccine, vol. 26, no. 10, pp. K29-K41, 2008.

[4] P. Giorgi Rossi, M. Benevolo, A. Vocaturo et al., "Prognostic value of HPV E6/E7 mRNA assay in women with negative colposcopy or CIN1 histology result: a follow-up study," PLOS One, vol. 8, no. 2, pp. 54-62, 2013.

[5] M. Guo, Y. Gong, M. Deavers et al., "Evaluation of a commercialized in situ hybridization assay for detecting human papillomavirus DNA in tissue specimens from patients with cervical intraepithelial neoplasia and cervical carcinoma," Journal of Clinical Microbiology, vol. 46, no. 1, pp. 274-280, 2008.

[6] M. M. Dabić, L. Hlupić, D. Babić, S. Jukić, and S. Seiwerth, "Comparison of polymerase chain reaction and catalyzed signal amplification in Situ hybridization methods for human papillomavirus detection in paraffin-embedded cervical preneoplastic and neoplastic lesions," Archives of Medical Research, vol. 35, no. 6, pp. 511-516, 2004.

[7] M. F. Evans, S. L. Mount, B. G. Beatty, and K. Cooper, "Biotinyltyramide-based in situ hybridization signal patterns distinguish human papillomavirus type and grade of cervical intraepithelial neoplasia," Modern Pathology, vol. 15, no. 12, pp. 1339-1347, 2002.

[8] J. D. Meissner, "Nucleotide sequences and further characterization of human papillomavirus DNA present in the CaSki, $\mathrm{SiHa}$ and HeLa cervical carcinoma cell lines," Journal of General Virology, vol. 80, no. 7, pp. 1725-1733, 1999.

[9] K. U. Petry, D. Schmidt, S. Scherbring et al., "Triaging Pap cytology negative, HPV positive cervical cancer screening results with p16/Ki-67 Dual-stained cytology," Gynecologic Oncology, vol. 121, no. 3, pp. 505-509, 2011.

[10] D. Schmidt, C. Bergeron, K. J. Denton, and R. Ridder, "p16/ki-67 dual-stain cytology in the triage of ASCUS and LSIL papanicolaou cytology: results from the European equivocal or mildly abnormal Papanicolaou cytology study," Cancer Cytopathology, vol. 119, no. 3, pp. 158-166, 2011.

[11] A. Tinelli, G. Leo, M. Pisanò et al., "HPV viral activity by mRNA-HPV molecular analysis to screen the transforming infections in precancer cervical lesions," Current Pharmaceutical Biotechnology, vol. 10, no. 8, pp. 767-771, 2009. 
[12] J. R. Landis and G. G. Koch, "The measurement of observer agreement for categorical data," Biometrics, vol. 33, no. 1, pp. 159-174, 1977.

[13] E. R. DeLong, D. M. DeLong, and D. L. Clarke-Pearson, "Comparing the areas under two or more correlated receiver operating characteristic curves: a nonparametric approach," Biometrics, vol. 44, no. 3, pp. 837-845, 1988.

[14] P. J. F. Snijders, A. J. C. van den Brule, and C. J. L. M. Meijer, “The clinical relevance of human papillomavirus testing: relationship between analytical and clinical sensitivity," Journal of Pathology, vol. 201, no. 1, pp. 1-6, 2003.

[15] R. Zappacosta, D. Caraceni, L. Ciccocioppo et al., "Is HPV-DNA testing a useful tool in predicting low-grade squamous intraepithelial lesion outcome? A retrospective longitudinal study," International Journal of Immunopathology and Pharmacology, vol. 23, no. 1, pp. 317-326, 2010.

[16] W. Qu, G. Jiang, Y. Cruz et al., "PCR detection of human papillomavirus: comparison between MY09/MY11 and GP5+/GP6+ primer systems," Journal of Clinical Microbiology, vol. 35, no. 6, pp. 1304-1310, 1997.

[17] M. Arbyn, P. Sasieni, C. J. L. M. Meijer, C. Clavel, G. Koliopoulos, and J. Dillner, "Chapter 9: clinical applications of HPV testing: a summary of meta-analyses," Vaccine, vol. 24, supplement 3, pp. S78-S89, 2006.

[18] M. F. Evans and K. Cooper, "Human papillomavirus integration: detection by in situ hybridization and potential clinical application," Journal of Pathology, vol. 202, no. 1, pp. 1-4, 2004.

[19] M. Montag, T. J. F. Blankenstein, N. Shabani, A. Brüning, and I. Mylonas, "Evaluation of two commercialised in situ hybridisation assays for detecting HPV-DNA in formalinfixed, paraffin-embedded tissue," Archives of Gynecology and Obstetrics, vol. 284, no. 4, pp. 999-1005, 2011.

[20] K. T. Kuo, C. H. Hsiao, C. H. Lin, L. Kuo, S. Huang, and M. Lin, "The biomarkers of human papillomavirus infection in tonsillar squamous cell carcinoma-molecular basis and predicting favorable outcome," Modern Pathology, vol. 21, no. 4, pp. 376-386, 2008.

[21] A. Luginbuhl, M. Sanders, and J. D. Spiro, "Prevalence, morphology, and prognosis of human papillomavirus in tonsillar cancer," Annals of Otology, Rhinology and Laryngology, vol. 118, no. 10, pp. 742-749, 2009.

[22] M. F. Evans, H. A. Aliesky, and K. Cooper, "Optimization of biotinyl-tyramide-based in situ hybridization for sensitive background-free applications on formalin-fixed, paraffinembedded tissue specimens," BMC Clinical Pathology, vol. 3, no. 1, pp. 1-17, 2003.

[23] M. F. Evans, A. Matthews, D. Kandil, C. S. Adamson, W. E. Trotman, and K. Cooper, "Discrimination of "Driver" and "Passenger" HPV in tonsillar carcinomas by the polymerase chain reaction, chromogenic in situ hybridization, and $\mathrm{p} 16^{\mathrm{INK} 4 \mathrm{a}}$ immunohistochemistry," Head and Neck Pathology, vol. 5, no. 4, pp. 344-348, 2011.

[24] G. M. Clifford, J. S. Smith, M. Plummer, N. Muñoz, and S. Franceschi, "Human papillomavirus types in invasive cervical cancer worldwide: a meta-analysis," British Journal of Cancer, vol. 88, no. 1, pp. 63-69, 2003.

[25] L. Kraus, T. Molden, R. Holm et al., "Presence of E6 and E7 mRNA from human papillomavirus types 16, 18, 31, 33, and 45 in the majority of cervical carcinomas," Journal of Clinical Microbiology, vol. 44, no. 4, pp. 1310-1317, 2006.
[26] S. de Sanjosé, M. Diaz, X. Castellsagué et al., "Worldwide prevalence and genotype distribution of cervical human papillomavirus DNA in women with normal cytology: a metaanalysis," Lancet Infectious Diseases, vol. 7, no. 7, pp. 453-459, 2007.

[27] G. Gallo, M. Bibbo, L. Bagella et al., "Study of viral integration of HPV-16 in young patients with LSIL," Journal of Clinical Pathology, vol. 56, no. 7, pp. 532-536, 2003.

[28] N. Murphy, M. Ring, C. C. B. B. Heffron et al., "p16 ${ }^{\mathrm{INK} 4 \mathrm{a}}$, CDC6, and MCM5: predictive biomarkers in cervical preinvasive neoplasia and cervical cancer," Journal of Clinical Pathology, vol. 58, no. 5, pp. 525-534, 2005.

[29] S. S. Wang, M. Trunk, M. Schiffman et al., "Validation of p16 $6^{\text {INK4a }}$ as a marker of oncogenic human papillomavirus infection in cervical biopsies from a population-based cohort in Costa Rica," Cancer Epidemiology Biomarkers and Prevention, vol. 13, no. 8, pp. 1355-1360, 2004.

[30] I. Tsoumpou, M. Arbyn, M. Kyrgiou et al., "p16 ${ }^{\text {INK4a }}$ immunostaining in cytological and histological specimens from the uterine cervix: a systematic review and meta-analysis," Cancer Treatment Reviews, vol. 35, no. 3, pp. 210-220, 2009.

[31] R. Klaes, T. Friedrich, D. Spitkovsky et al., "Overexpression of $\mathrm{p} 16^{\mathrm{INK} 4 \mathrm{a}}$ as a specific marker for dysplastic and neoplastic epithelial cells of the cervix uteri," International Journal of Cancer, vol. 92, no. 2, pp. 276-284, 2001.

[32] I. Tsoumpou, G. Valasoulis, C. Founta et al., "High-risk human papillomavirus DNA test and p16 $6^{\mathrm{INK} 4 \mathrm{a}}$ in the triage of LSIL: a prospective diagnostic study," Gynecologic Oncology, vol. 121, no. 1, pp. 49-53, 2011.

[33] K. J. Denton, C. Bergeron, P. Klement, M. J. Trunk, T. Keller, and R. Ridder, "The sensitivity and specificity of $16^{\text {INK4a }}$ cytology vs HPV testing for detecting high-grade cervical disease in the triage of ASC-US and LSIL Pap cytology results," American Journal of Clinical Pathology, vol. 134, no. 1, pp. 12-21, 2010.

[34] C. M. Martin and J. J. O'Leary, "Histology of cervical intraepithelial neoplasia and the role of biomarkers," Best Practice \& Research Clinical Obstetrics and Gynaecology, vol. 25, no. 5, pp. 605-615, 2011.

[35] J. L. Meyer, D. W. Hanlon, B. T. Andersen, O. F. Rasmussen, and K. Bisgaard, "Evaluation of $\mathrm{p} 16^{\mathrm{INK} 4 \mathrm{a}}$ expression in ThinPrep cervical specimens with the CINtec p16 ${ }^{\mathrm{INK} 4 \mathrm{a}}$ assay: correlation with biopsy follow-up results," Cancer, vol. 111, no. 2, pp. 83-92, 2007.

[36] M. del Pino, S. Garcia, V. Fusté et al., "Value of p16 ${ }^{\mathrm{INK} 4 \mathrm{a}}$ as a marker of progression/regression in cervical intraepithelial neoplasia grade 1," American Journal of Obstetrics and Gynecology, vol. 201, no. 5, pp. 488e1-488e7, 2009.

[37] L. M. Stewart, M. H. Einstein, W. K. Huh et al., "Guidelines for the management of abnormal cervical cancer screening tests and cancer precursors," Journal of Lower Genital Tract Disease, vol. 17, pp. 1-27, 2013. 


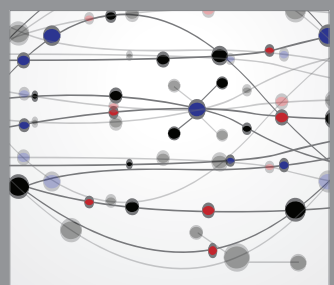

The Scientific World Journal
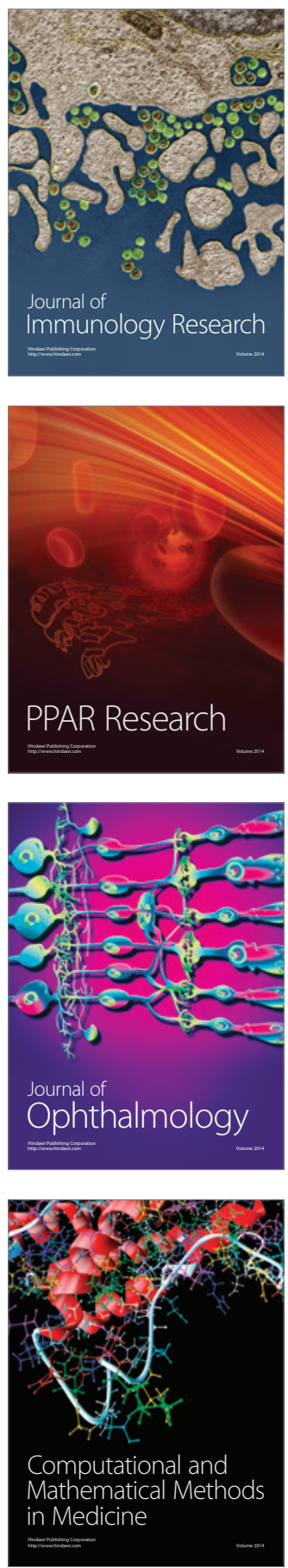



Gastroenterology

Research and Practice
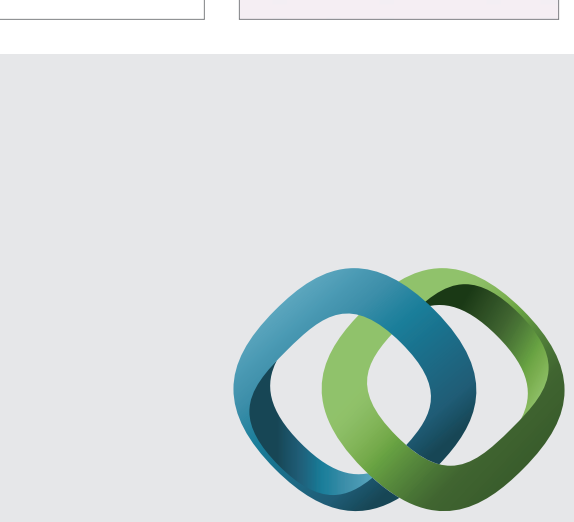

\section{Hindawi}

Submit your manuscripts at

http://www.hindawi.com
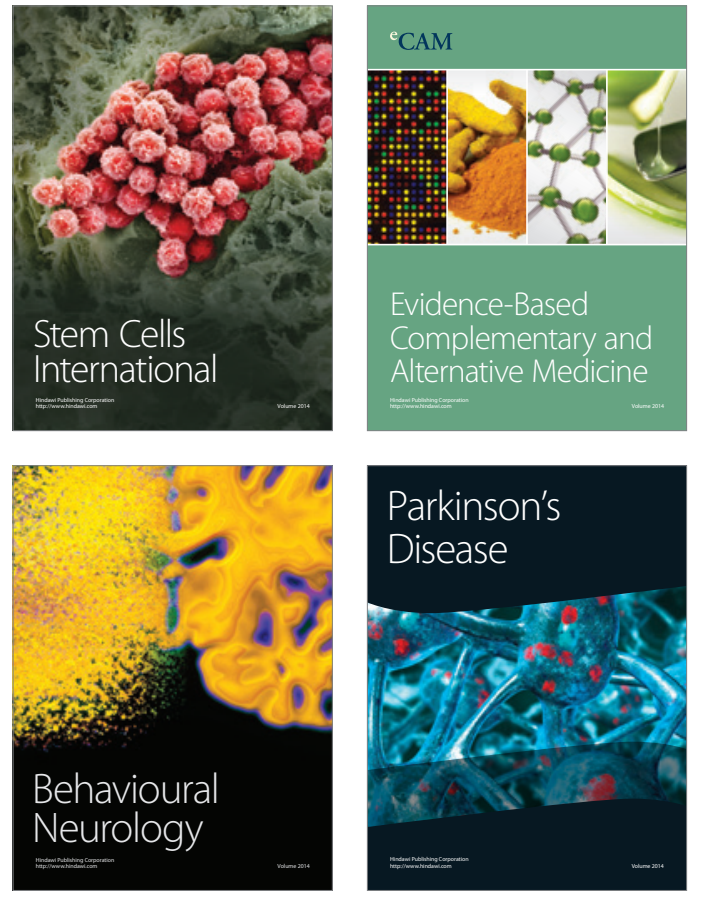
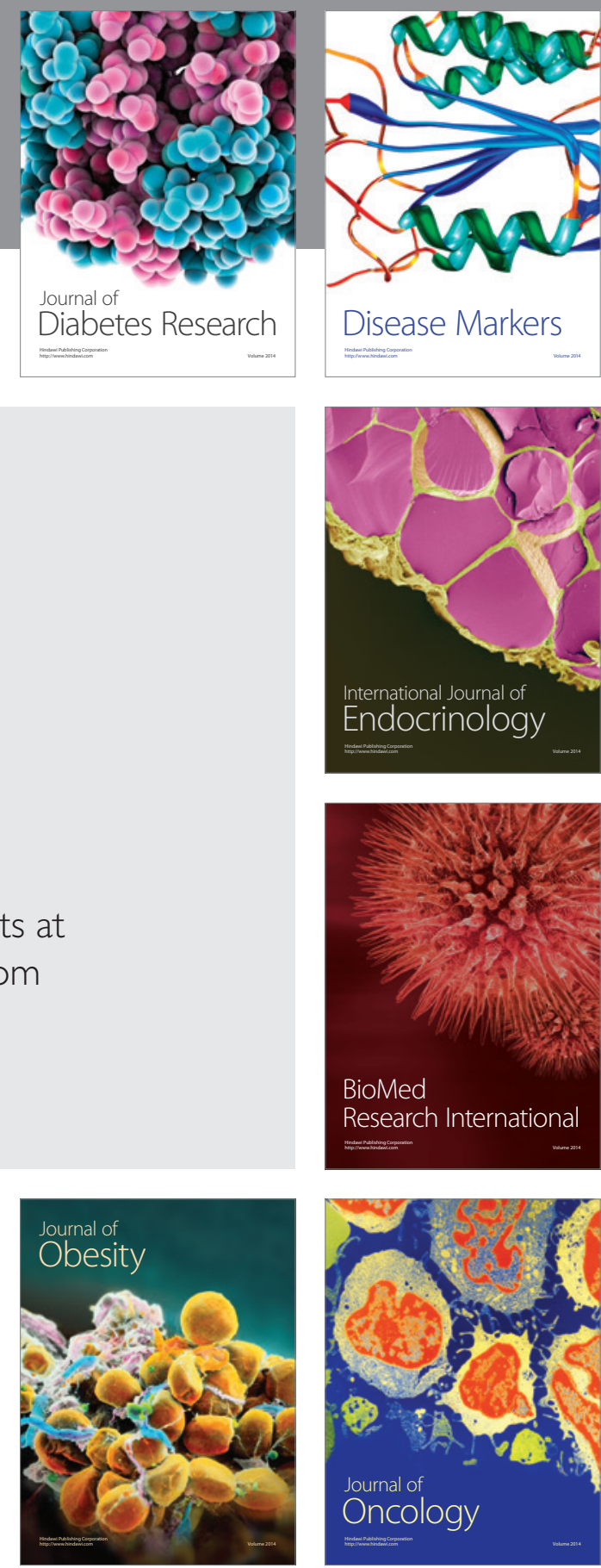

Disease Markers
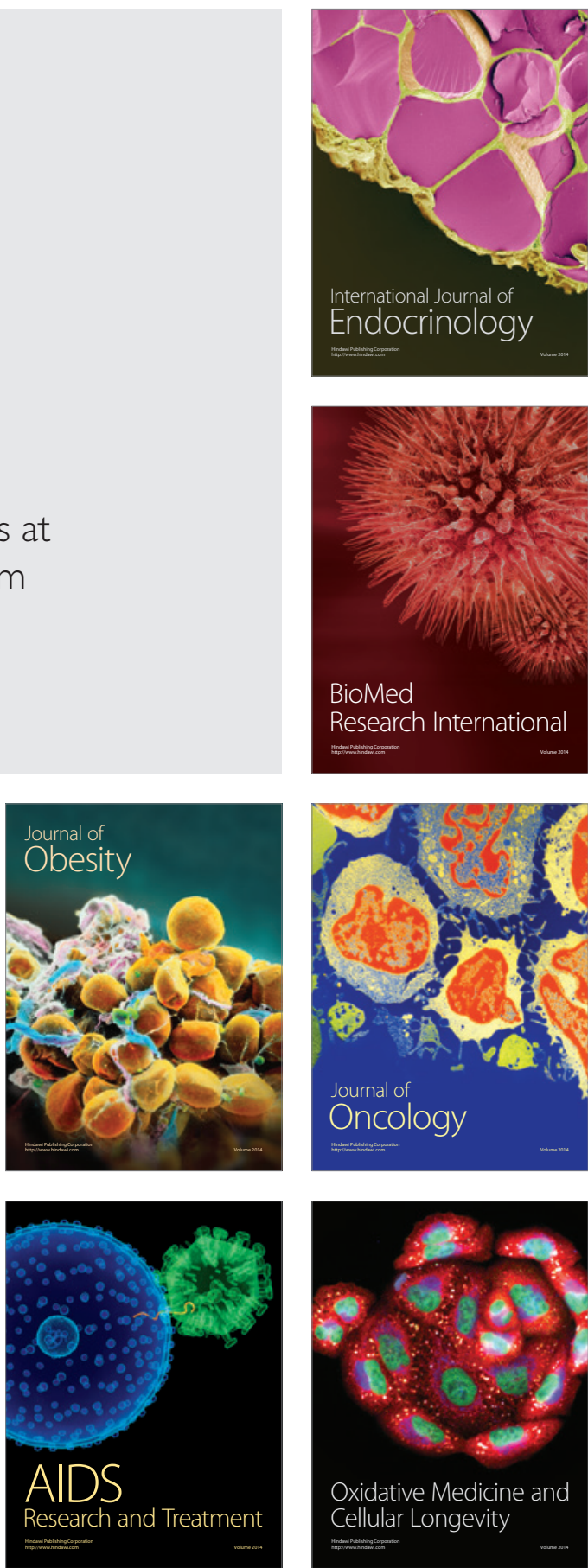